\title{
Nonlinear impact monitoring: 2-dimensional sampling
}

\author{
Giacomo Tommei ${ }^{1}$ \\ ${ }^{1}$ Department of Mathematics, University of Pisa, Via Buonarroti 2, 56126 Pisa, Italy \\ email: tommei@mail.dm.unipi.it
}

\begin{abstract}
When a new Near Earth Asteroid is discovered, it is important to know if there is the possibility of an impact with the Earth in the near future. In these last years second generation software for impact monitoring (CLOMON2 and SENTRY) have been developed and the performances have been significantly increased in comparison to the earlier, simpler and solitary system CLOMON. The two systems use the Line Of Variations (LOV) approach: they sample the LOV, an 1-dimensional subspace, to perform the sampling of the 6-dimensional confidence region. This approach is very useful when the confidence region is elongated and thin, that is an eigenvalue of the covariance matrix is much bigger than the others. When the observed arc is short $\left(1^{\circ}\right.$ or less), usually for asteroids observed for few nights, the confidence region is like a flat disk and we propose to use a 2-dimensional sampling. We triangulate the admissible region in the $(r, \dot{r})$ plane, using the nodes of triangulations as Virtual Asteroids (VAs). After orbit propagation we project the VAs and the triangulation on the Target Plane (TP) of a given epoch to study the existence of a Virtual Impactor (VI) and complex dynamical behaviors such as folds.
\end{abstract}

Keywords. Celestial mechanics, asteroids, methods: numerical

\section{Introduction}

When an asteroid has just been discovered, its orbit is weakly constrained by the available observations spanning a short arc. In many cases a nominal orbital solution exists, but other sets of orbital elements, that correspond to RMS of the residuals not significantly above the minimum, are acceptable as solutions. This situation can be described by defining a confidence region $Z(\sigma)$ in the orbital elements space such that initial orbital elements belong to $Z(\sigma)$ if the penalty (increase in the target function) does not exceed some threshold depending upon the parameter $\sigma$.

In many applications we need to consider the set of the orbits with initial conditions in the confidence region as a whole. In impact monitoring we may need to predict some future close approach or even an impact, and this for all possible orbits, that is for every solution not resulting in too large residuals. Since the dynamic model for asteroid orbits is not integrable there is no way to compute all the solutions for some time span in the future. We can only compute a finite number of orbits by numerical integration. There are several methods to perform a sampling of the confidence region. In this paper we explore the geometrical sampling, reviewing the Line Of Variations (LOV) approach introduced by Milani (1999) (Section 2) and proposing a new 2-dimensional sampling for cases in which the LOV approach is not satisfactory (Section 3). In the last Section we show as this new sampling could be used to study complex dynamical behaviors such as folds. 


\section{The LOV approach in impact monitoring}

The LOV is a 1-dimensional segment of a curved line in the 6-dimensional space of initial conditions. There is a number of different ways to define and compute the LOV, but the general idea is that a segment of this line is a kind of spine of the confidence region. Both CLOMON2 and SENTRY, the two independent systems of impact monitoring, use the LOV approach. The LOV is sampled at regular intervals obtaining an ordered set of Virtual Asteroids (VAs). In this way it is possible to interpolate between two consecutive VAs to prove the existence of a closest approach around a given encounter date. Using iterative algorithms it is possible to find the minimum approach distance and eventually a Virtual Impactor (VI) (Milani et al. (2004b)).

In Milani (1999) the LOV was defined as the solution of an ordinary differential equation of the first order, with the nominal least squares solution as initial condition, and the vector field defined by the weak direction of the covariance matrix, the eigenvector relative to the biggest eigenvalue of the matrix. Such differential equation is unstable, in particular when there is a largely dominant weak direction, the case when the LOV is most useful. Thus a corrective step was introduced, based upon differential corrections constrained on the hyperplane perpendicular to the weak direction. Milani et al. (2004c) have formulated a new definition which does not require the presence of a nominal solution. The LOV, in fact, is defined as a set of points in which the gradient of the cost function is in the weak direction. All the LOVs used in this paper to produce numerical results are obtained with this constrained differential correction algorithm.

There is an important fact to remark: the eigenvalues of covariance matrix are not invariant under a general coordinate change (only for an orthonormal one), so the LOV depends upon the coordinates used for initial orbit determination. Then it is possible to compute several LOVs and the choice of one of these to use in impact monitoring is not always a simple issue.

The next two figures show different LOVs for two Near Earth Asteroids (NEAs) in the plane $(r, \dot{r})$ with $\dot{r}$ scaled by the Gaussian constant. For each coordinates system (Cartesian, Attributable, Equinoctial, Cometary) we trace both the LOV (marked with 1), sampled by 41 VAs for $\sigma \in[-1,1]$, and the second LOV (marked with 2 ), obtained using the eigenvector relative to the second largest eigenvalue of the covariance matrix.

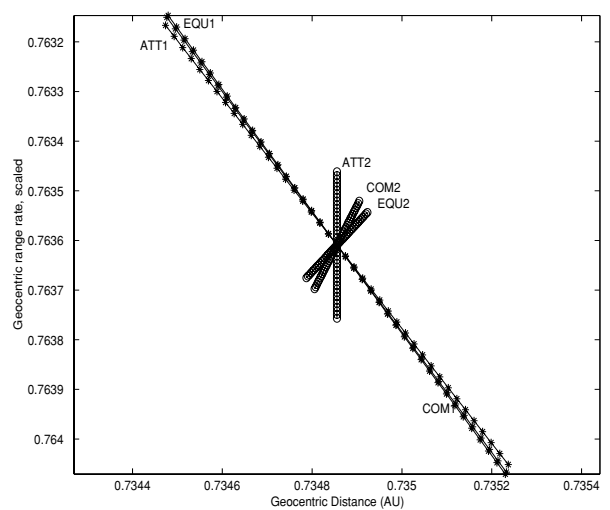

Figure 1. Milani et al. (2004c). LOVs in different coordinates for the asteroid $2002 \mathrm{NT}_{7}$ with the first 113 observations. The Cartesian and Attributable LOVs coincide so only the Attributable one is depicted.

Figure 1 shows the comparison of LOVs for asteroid $2002 \mathrm{NT}_{7}$ when the 113 available observations were spanning 15 days forming an arc of about $9^{\circ}$. In this case the LOVs 
are very similar and the choice of one instead of another to use in impact monitoring does not produce significant changes in results. If we imagine the ellipse of confidence in this plane, having as axes LOV and second LOV, we note that it is very elongated and thin.

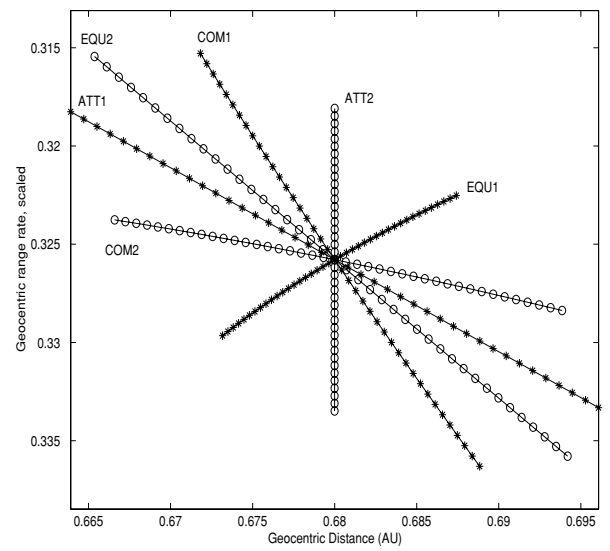

Figure 2. Milani et al. (2004c). LOVs in different coordinates for the asteroid $2004 \mathrm{FU}_{4}$ with the first 17 observations. The Cartesian and Attributable LOV coincide so only the Attributable one is depicted.

Figure 2 shows the LOVs for asteroid $2004 \mathrm{FU}_{4}$ when the 17 available observations were spanning 3 days forming an arc of about $1^{\circ}$. The dependence of the LOV on the coordinates is very strong here. Note that the LOV of the Attributable or Cartesian elements is near the second LOV of the Equinoctial elements. In this case the choice of a LOV as sampling of the confidence region to use in impact monitoring is not simple. In fact, with these data, using the LOV computed in Cartesian elements CLOMON2 did not find a VI in 2010, while using Equinoctial, or SENTRY, using Cometary, did find it. The ellipse of confidence in this figure is like a flat disk, so the idea is to switch to a new kind of sampling, to perform a better analysis of the geometric structure of this 2-dimensional subset.

\section{2-dimensional sampling}

This new approach does not require the presence of a preliminary orbit, but only the observations. Starting from these it is possible to create an attributable which consists of a reference time (the mean of observations times), two average angular coordinates and two corresponding angular rates for the reference time.

Following the work of Milani et al. (2004a), we define an admissible region and we triangulate it using the Delaunay triangulation. Figure 3 shows the triangulated admissible region for the asteroid $2004 \mathrm{FU}_{4}$ with the first 17 observations, the case analyzed in the previous Section. Each node of the triangulation is used as VA and it is propagated to a target time recording all the close approaches to the Earth. Then, after a partition in showers, depending upon the time of close approaches, each VA is projected on the usual Target Plane (TP) of Öpik theory. Figure 4 shows the TP of first close approach of 2004 $\mathrm{FU}_{4}$ after the discovery, with the triangulation obtained joining the nodes which belong to the same triangle in the original triangulation.

After some close approaches the geometry on the TP becomes very complicated. Figure 5 shows the TP of 2010 for $2004 \mathrm{FU}_{4}$ with the same data. The triangulation 


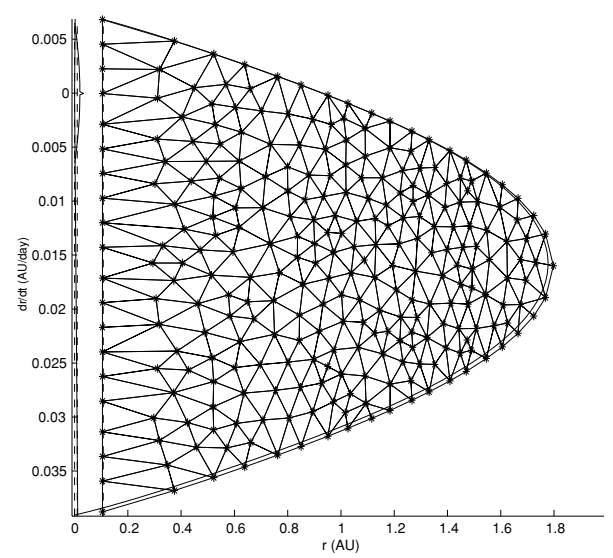

Figure 3. Triangulation of the admissible region in the plane $(r, \dot{r})$ for the asteroid $2004 \mathrm{FU}_{4}$ with the first 17 observations.

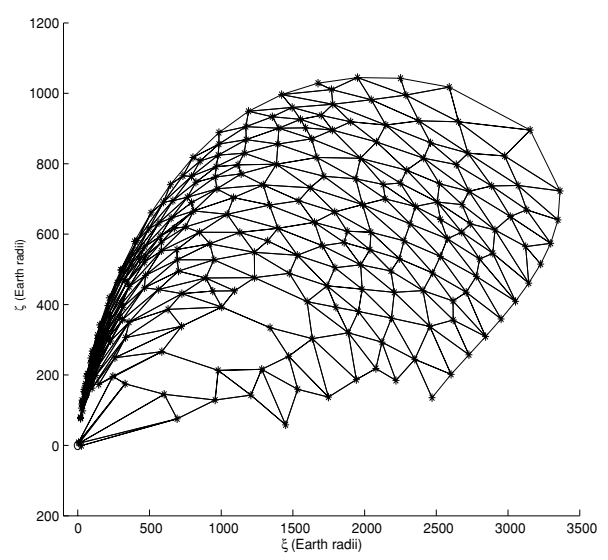

Figure 4. Triangulation on the TP of the first close approach in 2004 for the asteroid 2004 $\mathrm{FU}_{4}$ with the first 17 observations.

appears very complicated but the remarkable fact is that the Earth is in at least one triangle, so the presence of a VI could not be excluded. In this figure it is also possible to note folds, complex behaviors derived from returns to close approaches that will be explained in the next Section.

\section{An example of complex dynamical behavior: the folds}

A return to a close approach, after a previous encounter (after the time of the initial conditions), is mathematically represented as a map between the TP of the first encounter and the TP of the second one. The behavior of differentiable maps $\mathbf{R}^{2} \rightarrow \mathbf{R}^{2}$ has been studied by Witney (1950). He proved that generically (for a set of maps dense in the function space of all differentiable maps) the points of the first plane which are singular (that is, at which the differential of the map is not invertible) form smooth curves on the first plane and belong to only two geometrically distinct types: the folds and the cusps. A fold is such that the image on the second plane of the curve of singular points is a locally regular curve, while a cusp of the 2-dimensional map corresponds to a cusp (reversal of the tangent vector) on the image on the second TP of the curve of the singular 


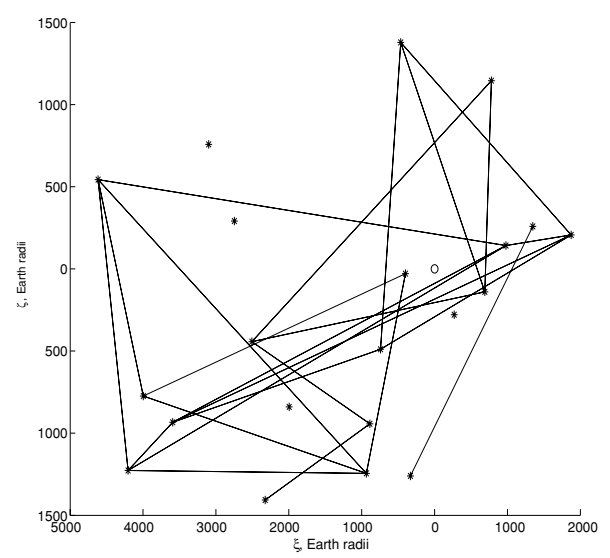

Figure 5. Triangulation on the TP of 2010 for the asteroid $2004 \mathrm{FU}_{4}$ with the first 17 observations.

points. The fold points are such that the map can be locally transformed, by suitable differentiable coordinate changes (in both planes), into the normal form

$$
\xi^{\prime \prime}=\xi \quad \zeta^{\prime \prime}=\zeta^{2} .
$$

In a intuitive way, the first TP folds along the $\zeta=0$ fold line and covers twice the $\zeta^{\prime \prime} \geqslant 0$ half plane. For the purpose of searching for VIs it is therefore essential to know if the fold is in the direction from the fold line towards the Earth or away from it. The role of cusps in impact monitoring will be the subject of future research.

Figure 6 shows an interrupted return, an example of fold obtained by a 1-dimensional sampling, using the LOV. The sequence of VAs approaches the Earth (the origin of the reference system) and then turns before reaching it.

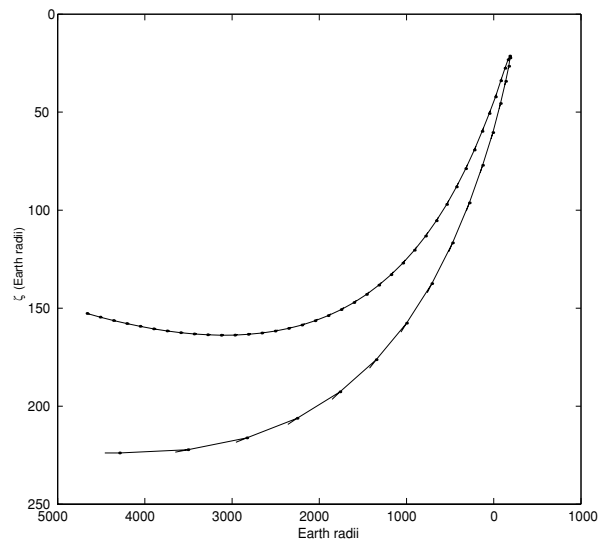

Figure 6. Interrupted return on the TP of 2025 for the asteroid 1994 UG.

In Figure 7 it is possible to appreciate the real 2-dimensional nature of a fold: a triangle folds and covers other triangles, but in such way that the Earth (depicted with a circle, not to scale) is protected by the fold and there is no VI. 


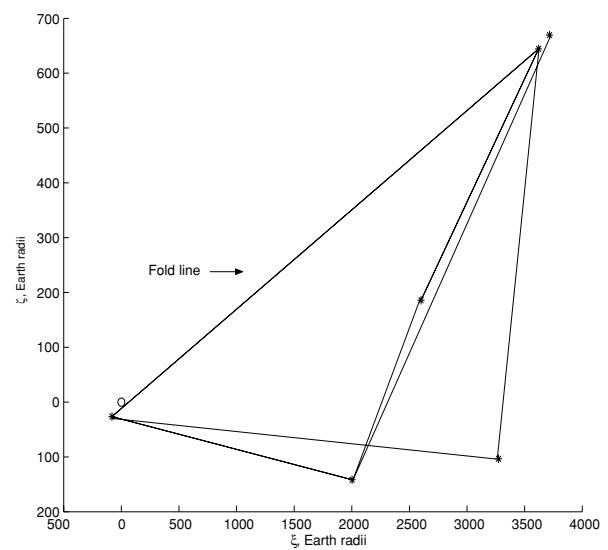

Figure 7. Example of fold on the TP of 2005 for the asteroid $2004 \mathrm{FU}_{4}$ (first 7 observations) found using a 2-dimensional sampling.

\section{Conclusions and future work}

The LOV approach works well when there is a largely dominant weak direction and it is possible to use the geometric properties of a string. When the confidence region has a 2-dimensional structure, it is useful to switch to the new approach explained in the paper. In practice this happens when the arc is very short ( $1^{\circ}$ for example, as for 2004 FU4). The goal of this kind of geometrical sampling is to be able to analyze asteroids with few nights of observations. We would like to know if they could have either direct impacts (impacts before the next close approach) or VIs as returns after some close encounters. This new approach could also be useful to handle complex behaviors, such as folds, which could give us information about the geometry on the TP.

Next step will be to focus on the part of the triangulation which has acceptable residuals, using differential corrections in the 4-dimensional space orthogonal to the 2dimensional triangulated manifold. Then, the idea is to create recursive algorithms to improve the triangulation obtained, defining criteria to detect VIs, to extrapolate an estimate of impact probability.

\section{Acknowledgments}

I would like to acknowledge A. Milani, who proposed the problem, for his suggestions, G.F. Gronchi, G.B. Valsecchi and S.R. Chesley for useful discussions.

\section{References}

Milani, A. 1999, Icarus, 137, pp. 269-292

Milani, A., Gronchi, G.F., De’Michieli Vitturi, M. \& Knežević, Z. 2004a, Cel. Mech. Dyn. Astron., in press

Milani, A., Chesley, S.R., Sansaturio, M.E., Tommei, G. \& Valsecchi, G.B. 2004b, Icarus, in press

Milani, A., Sansaturio, M.E., Tommei, G., Arratia, O. \& Chesley, S.R. 2004c, Astron. Astrophys., in press 